\title{
Génesis y evolución de la artroplastia de cadera
}

\section{Genesis and Evolution of Hip Arthroplasty}

Recibido: 15/07/2020 | Aceptado: 14/08/2020

Hernando Gaitán LeE

Departamento de Ortopedia y Traumatología de la Facultad de Medicina, Pontificia Universidad Javeriana-Hospital Universitario San Ignacio, Colombia ORCID: https://orcid.org/0000-0003-4913-7764 Diana Sarmiento-Osorio

Médica cirujana, Pontificia Universidad Javeriana, Colombia ORCID: https://orcid.org/0000-0002-7640-7718

a Autora de correspondencia: dsarmiento@javeriana.edu.co

Cómo citar: Gaitán Lee H, Sarmiento-Osorio D. Génesis y evolución de la artroplastia de cadera. Univ. Med. 2021;62(1). https://doi.org/10.11144/Javeriana. umed62-1.geac

\section{RESUMEN}

Los remplazos articulares de cadera configuran una serie de técnicas quirúrgicas ampliamente estudiadas que proveen resultados satisfactorios en términos de mejoría del dolor, funcionalidad, independencia para actividades básicas y calidad de vida en los pacientes adultos jóvenes y mayores con patología articular de la articulación. A lo largo de la historia, los cirujanos ortopedistas han realizado intervenciones desde osteotomías intertrocantéreas, pasando por interposiciones en la articulación, hasta el desarrollo de prótesis de alta tecnología con descripciones de diferentes abordajes y técnicas quirúrgicas. Este artículo recorre la historia de los remplazos articulares; es una síntesis de la evolución de los pilares de la cirugía de remplazos articulares que permite al lector entender los principios que fundamentan la tecnología y técnicas vigentes en la artroplastia de cadera.

Palabras clave

remplazo total de cadera; artroplastia; remplazo total de cadera cementado; remplazo total de cadera no cementado; osteotomía; prótesis.

\begin{abstract}
Total hip replacement includes a series of surgical techniques widely studied that provide patients satisfactory outcomes in terms of pain relief, limb and gait functionality, independence for basic activities and quality of life in young adults and geriatric patients diagnosed with hip pathology. Throughout history, orthopedic surgeons have performed procedures that range from intertrochanteric osteotomy, articular interposition of a variety of materials, until developing high technology prosthesis as well as describing different surgical techniques. This manuscript provides a journey through the history of total hip replacements; brings a synthesis of the evolution of the key concepts in total hip replacements that allows their reader to understand the principles that developed technology and surgical techniques used in total hip arthroplasty.

Keywords

total hip replacement; arthroplasty; cemented hip replacement; uncemented total hip replacement; osteotomy; protheses.
\end{abstract}




\section{Introducción}

El dolor articular en la cadera es un síntoma prevalente y una causa significativa de limitación funcional en el adulto joven y el adulto mayor. Estudios de corte transversal en Estados Unidos han reportado la prevalencia de coxalgia hasta del 19,2\% en los pacientes adultos mayores de 65 años (1). Otras estimaciones han documentado que hasta el $40 \%$ de los adultos mayores de 64 años en Inglaterra manifiestan dolor articular en cadera o rodilla secundarios a patología inflamatoria $(1,2,3)$; es un síntoma detrás del cual se esconden un amplio espectro de condiciones clínicas, entre estas osteoartrosis de la cadera, secuelas por deformidad congénita, secuelas de osteonecrosis, lesiones traumáticas, infecciones osteoarticulares, entre otros, por lo cual la cirugía ortopédica ha evolucionado a lo largo de los años para proveer a los pacientes mejoría clínica en términos de dolor, movilidad, discapacidad física y calidad de vida $(3,4)$.

Estimaciones realizadas en 2010 por el estudio de carga de enfermedad global, conducido en la Universidad de Washington junto con el Instituto de Métrica en Salud y Evaluación (5), sugieren que la osteoartrosis de cadera, entre las 291 condiciones reportadas, ocupan el puesto 11 como contribuyente global de discapacidad y el puesto 38 en carga de enfermedad por años de vida ajustados a discapacidad. Por ello, no debe subestimarse la importancia de su manejo integral en ortopedia, parte del cual es provisto por los remplazos articulares de cadera $(5,6)$.

Observaciones, a partir de estudios de costos, calcularon que cada remplazo articular de cadera sin complicaciones en Australia entre 2004 y 2005, tuvo un costo aproximado de 10.000 dólares americanos (7). Luego, en 2011, estudios de costos australianos también lograron demostrar una relación de costo-efectividad incremental con marcada favorabilidad en torno a los remplazos articulares de cadera entre 8500 y 10.000 dólares por año de vida ajustado a discapacidad, cuya relevancia no es despreciable para los proveedores de salud (8).

Los remplazos articulares de cadera no solo representan un alivio en términos de carga de enfermedad para los pacientes; además, tienen una implicación en costos para los proveedores de salud, que no debe subestimarse. El dominio de la artroplastia de cadera comprende un tema de interés para la comunidad de ortopedistas, y el recuento histórico de los pilares que constituyen actualmente los cimientos de los remplazos articulares traídos al contexto de las técnicas a la vanguardia y en relación con su contexto en Colombia y en el Departamento de Ortopedia de la Pontificia Universidad Javeriana motivaron la redacción de este artículo.

\section{Evolución de los remplazos articulares}

Los remplazos articulares de la cadera datan de 1826, cuando el Dr. John Rea Barthon, ortopedista del Hospital de Pensilvania, llevó a cabo una osteotomía intertrocantérica sin anestesia en tan solo siete minutos en un paciente (figura 1). Se trataba de un marinero de 21 años de edad con anquilosis de la cadera que inició después de una caída de aproximadamente dos metros de altura mientras se encontraba trabajando en $1825(9,10,11)$.

\section{Figura 1.}

Planeamiento quirúrgico del Dr. John Rea Barthon

(9)

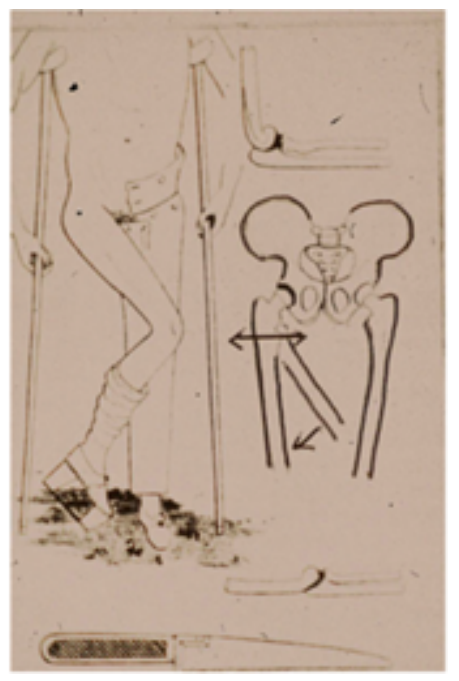

Pasados 20 días de la intervención, el Dr. Barthon, manipuló y movió la extremidad, por lo cual el foco de la osteotomía se diferenció 
como tejido fibroso y posteriormente generó seudoartrosis. En el postoperatorio, el paciente recuperó la marcha asistido de un bastón tres meses después de la intervención; sin embargo, pasados seis años, el paciente perdió nuevamente la funcionalidad y el movimiento de la extremidad. A pesar de lo anterior, el Dr. Barthon introdujo un concepto importante y revolucionario en la ortopedia, devolver a un paciente la movilidad de una articulación anquilosada. Este sería el pilar que hoy ha evolucionado hasta artroplastias seguras, exitosas y eficientes $(9,10,11,12)$.

Después de los trabajos del Dr. Barthon, los cirujanos ortopedistas se encontraron frente al reto de reducir la recurrencia de los síntomas y, aún más importante, reducir la mortalidad, que llegaba hasta el $50 \%$ en los pacientes que eran llevados a osteotomías (8).

En 1840, el Dr. Carnochan, en Nueva York, fue el primer cirujano en introducir el concepto de la interposición de materiales en la articulación. Convencido de que la articulación podía ser remplazada empezó a introducir bloques de madera y luego utilizó un amplio espectro de materiales biológicos, incluyendo vejiga de cerdo y otras especies. Posterior al Dr. Carnochan, los médicos franceses Auguste Stanislas Verneuil y Léopold Ollier, en París y Lyon, respectivamente, desarrollaron mucho más el concepto de la artroplastia por interposición de tejidos blandos; sin embargo, sus esfuerzos dieron pobres resultados, dado que no fijaban los tejidos interpuestos a las superficies óseas subyacentes, por lo que sus intervenciones fallaron. A pesar de esto, fue un gran avance para el desarrollo de la artroplastia por interposición $(9,12,13)$.

Posteriormente, la cirugía ortopédica evolucionó sobre el eje de la interposición articular de diferentes materiales. Uno de los cirujanos que más describió cirugías haciendo uso de interposición de materiales orgánicos e inorgánicos fue el cirujano checo Vitezslav Chlumsky, quien operó pacientes en Alemania entre 1880 y 1890 . Para entonces era claro que la osteotomía aislada terminaba en inestabilidad, dolor y recurrencia de la limitación funcional, por lo cual era imperativo remplazar la articulación $(12,14)$.

El profesor Temístocles Gluck es el gran referente de la fijación de prótesis en la cadera. En 1891, condujo la producción de una prótesis de mármol en forma de bola y zócalo que remplazaría las superficies articulares del acetábulo y cabeza femoral que se fijan al hueso subyacente por medio de tornillos niquelados y dio los primeros avances en el uso de cemento óseo para mejorar la fijación de los componentes a las superficies óseas $(12,15,16)$. A pesar de que sus remplazos articulares eran exitosos, a largo plazo sus resultados fueron inaceptables, dado que sus pacientes sufrían de infección crónica en el sitio operatorio con falla de la prótesis. Sin embargo, el Dr. Gluck realizó aportes valiosos al desarrollo de la tecnología de remplazos articulares, dado que describió que los materiales utilizados debían ser biocompatibles y fijarse a las superficies óseas subyacentes (12).

La evolución de los hallazgos de todos los cirujanos ortopedistas se consolidaron en los años sesenta, cuando el Dr. Charnley introdujo tres conceptos clave que revolucionaron los remplazos articulares y mejoraron exponencialmente la supervivencia de los pacientes, que se reportó entre el $77 \%$ y el $81 \%$ pasados 25 años de la intervención (figura 2); por esto, los remplazos articulares de la cadera pasaron a llamarse la cirugía del siglo $(17,18,19)$. Las contribuciones del Dr. Charnley incluyeron laartroplastia de baja fricción, que se logra asegurando que el componente de la cabeza femoral sea del menor radio posible respecto al componente acetabular, de manera que produce una fuerza de torque más baja en la copa del acetábulo. Además, el Dr. Charnley popularizó el uso de cemento óseo de polimetilmetacrilato (PMMA) para la fijación de la prótesis a la superficie ósea subyacente y, por último, el uso de polietileno de alta densidad para la copa acetabular $(17,20)$. 


\section{Figura 2.}

Radiografía postoperatoria de un paciente de 26 años llevado a artroplastia de baja fricción de Charnley (17)

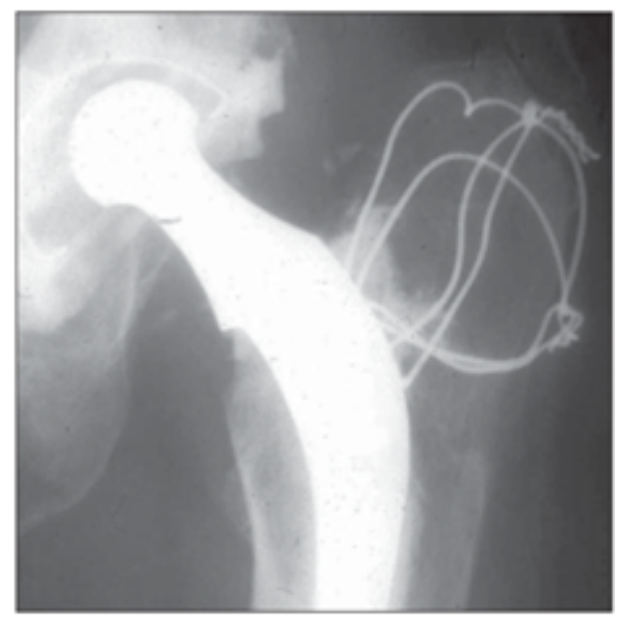

Sin embargo, llamaba la atención el aflojamiento temprano del vástago cementado. En 1970, investigadores encontraron que el cemento óseo generaba una reacción de fagocitosis al PMMA y posterior osteólisis, por lo que terminaba en aflojamiento aséptico de las prótesis $(21,22,23)$. El estudio subsecuente del aflojamiento de las prótesis permitió aclarar que este no se atribuía a la fagocitosis del PMMA, sino que se trataba de la fagocitosis del polietileno, que generaba osteólisis, migración en la interfase periprotésica de los detritos particulados y terminaba en el aflojamiento subsecuente de los componentes. De ahí data el inicio de investigaciones en tecnología de alta calidad del polietileno, que disminuyen el desgaste y el aflojamiento $(24,25,26)$ (figura 3).

\section{Figura 3.}

Radiografía tomada en un paciente a los 83 meses postoperatorios. Osteólisis por polietileno documentada en el fémur proximal y periacetabular (24)

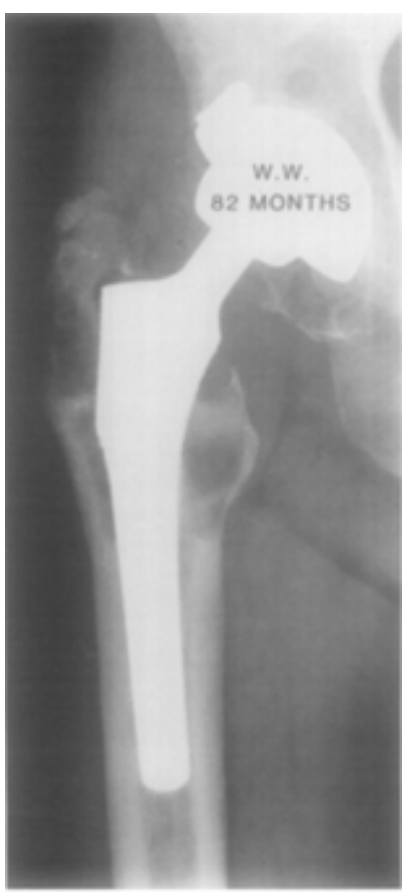

De forma simultánea, inició el desarrollo de los remplazos articulares no cementados, que se utilizan actualmente y cuya tecnología en materiales, recubrimiento, características de las prótesis, así como los diferentes abordajes quirúrgicos y su estandarización, comprende toda una evolución por medio de la ingeniería biomédica y la cirugía ortopédica (17).

El Departamento de Ortopedia y Traumatología de la Pontificia Universidad Javeriana y el Hospital Universitario San Ignacio han estado a la vanguardia de este procedimiento en Colombia (27). El doctor Eusebio Cadena llevó a cabo el primer remplazo total de cadera del país en 1970 (28), y posteriormente publicó en los años noventa su experiencia en la Revista Colombiana de Ortopedia y Traumatología, después de realizar un total de 188 remplazos articulares en el Hospital Universitario San Ignacio entre diciembre de 1987 y junio de 1991, en la que favoreció el uso de los remplazos articulares cementados a pesar de su 
impopularidad, atribuidos en ese entonces al aflojamiento secundario al uso de cemento óseo (teoría que fue aclarada posteriormente) (29). Desde ese momento, el doctor Eusebio Cadena ha permanecido como uno de los renombrados profesores y cirujanos de cadera (30).

En la actualidad, el remplazo total de cadera es considerado uno de los procedimientos más confiables y exitosos no solo en la ortopedia, sino en general en medicina $(4,31)$. La literatura sobre el tema reporta tasas de satisfacción mayores al $93 \%$ (32), mejoría significativa en la función $(33,34)$ y tasas de sobrevida cercanas al $90 \%$ a 15 años y al $77 \%$ a los 25 años (35). El número de procedimientos ha aumentado sustancialmente en los últimos años y las proyecciones sugieren que podría llegar hasta las 635.000 cirugías al año en el 2030 (36).

El futuro de la artroplastia de cadera se plantea fascinante. La cirugía guiada por navegación y la utilización de robótica prometen brindar mejor precisión y aún mayor confiabilidad al procedimiento $(37,38,39)$. Estos avances ya se encuentran en funcionamiento en algunos centros altamente especializados; pero con el tiempo, y una vez su utilización tenga una accesibilidad creciente, permitirá difundir aún más su uso $(37,40)$.

\section{Conclusiones}

La patología articular de la cadera comprende un problema altamente prevalente que impacta al adulto joven y adulto mayor en términos de dolor, movimiento y calidad de vida. Los principios descritos en este artículo, a través de la narración histórica de diferentes ortopedistas, permiten un acercamiento retrospectivo de la artroplastia de cadera que actualmente cuenta con estrategias de planeamiento quirúrgico de muy alta tecnología, así como la extensa variedad de componentes protésicos y abordajes quirúrgicos que han evolucionado a lo largo de los años.

El remplazo total de cadera es posiblemente la cirugía más revolucionaria de los últimos tiempos, en términos de mejoría en dolor y función para los pacientes. El conocimiento a profundidad de los pilares de la artroplastia de cadera y su origen son de valor para el ortopedista y especialidades afines, ya que fundamentan la práctica clínica vigente.

\section{Conflicto de intereses}

Los autores se declaran libres de conflictos de intereses.

\section{Agradecimientos}

Al doctor Efraim Leal García y al doctor Francisco Castro, por su disposición y comentarios al manuscrito.

\section{Referencias}

1. Dawson J, Linsell L, Zondervan K, Rose P, Randall T, Carr A, et al. Epidemiology of hip and knee pain and its impact on overall health status in older adults. Rheumatology (Oxford). 2004;43(4):497-504. https://doi.o rg/10.1093/rheumatology/keh086

2. Pålsson A, Kostogiannis I, Lindvall $\mathrm{H}$, Ageberg E. Hip-related groin pain, patient characteristics and patient-reported outcomes in patients referred to tertiary care due to longstanding hip and groin pain: a cross-sectional study. BMC Musculoskelet Disord. 2019;20(1):432. htt ps://doi.org/10.1186/s12891-019-2794-7

3. Zhang W, Moskowitz RW, Nuki G, Kwoh K, Lohmander LS, Tugwell P. OARSI recommendations for the management of hip and knee osteoarthritis, Part II: OARSI evidencebased, expert consensus guidelines. Osteoarthritis Cartilage. 2008;16(2):13762. https://doi.org/10.1016/j.joca.2007.12. 013

4. Lee JM. The current concepts of total hip arthroplasty. Hip Pelvis. 2016;28(4):191200. https://doi.org/10.5371/hp.2016.28.4. 191 
5. Institute for Health Metrics and Evaluation (IHME). GBD Compare Data Visualization [internet]. Seattle, WA: IHME, University of Washington; 2018 [citado 2020 ago 11]. Disponible en: http://vizhub.healthdata.org /gbd-compare.

6. Cross M, Smith E, Hoy D, Nolte S, Ackerman I, Fransen M, et al. The global burden of hip and knee osteoarthritis: estimates from the global burden of disease 2010 study. Ann Rheum Dis. 2014;73(7):1323-30. https://d oi.org/10.1136/annrheumdis-2013-204763

7. March LM, Barcenilla AL, Cross MJ, Lapsley HM, Brooks PM. Costs and outcomes of total hip and knee joint replacement for rheumatoid arthritis. Clin Rheumatol. 2008;27:1235-42. https://doi.org/10.1007/s 10067-008-0891-3

8. Higashi H, Barendregt JJ. Cost-effectiveness of total hip and knee replacements for the Australian population with osteoarthritis: discrete-event simulation model. PLoS One. 2011;6(9):e25403. https://doi.org/10. 1371/journal.pone.0025403

9. Hernigou P. Earliest times before hip arthroplasty: from John Rhea Barton to Themistocles Glück. Int Orthop. 2013;37(11):2313-8. https://doi.org/10.100 7/s00264-013-2004-4

10. Barton JR. On the treatment of anchylosis, by the formation of artificial joints. Lond Med Phys J. 1827;3(14):138-50.

11. Barton JR. The Classic: on the treatment of ankylosis by the formation of artificial joints. Clin Orthop Relat Res. 2007;456:9-14. https://doi.org/10.1097/blo. Ob013e31803254fd

12. Gómez PF, Morcuende JA. Early attempts at hip arthroplasty--1700s to 1950 s. Iowa Orthop J. 2005;25:25-9.

13. Ollier L. Traité des résections et des opérations conservatrices: qu'on peut pratiquer sue le système osseux. Vol. 2. París: G. Masson; 1889.

14. Chlumsky V. Continued Centralblatt für orthopaedische Chirurgie und mechanik from 1887-1890. Zentralblatt für orthopaedische Chirurgie; 1896.
15. Rang M. Anthology of orthopaedics. Edinburgh: Churchill Livingstone; 1966.

16. Glück, T. Referat über die durch das moderne chirurgische experiment gewonnenen positiven resultaten, betreffende die naht und den ersatz von defekten höherer gewebe, sowie über die verwerthung resorbirbarer und lebendiger tampons in der chirurgie. Arch Klin Chir. 1981;41:187-239.

17. Learmonth ID, Young C, Rorabeck C. The operation of the century: total hip replacement. Lancet. 2007;370(9597):1508-19. https://doi.org/10 .1016/S0140-6736(07)60457-7

18. Berry DJ, Harmsen WS, Cabanela ME, Morrey BF. Twenty-five-year survivorship of two thousand consecutive primary Charnley total hip replacements: factors affecting survivorship of acetabular and femoral components. J Bone Joint Surg Am. 2002;84(2):171-7. https://doi.org/10.2106/0 0004623-200202000-00002

19. Convery FR. Hip arthroplasty. Edited By H. C. Amstutz. New York: Churchill Livingstone, 1991. J Orthop Res. 1992;10:601. https://doi.org/10.1002/jor.11 00100417

20. Charnley J. Arthroplasty of the hip. A new operation. Lancet. 1961;1(7187):112932. https://doi.org/10.1016/s0140-6736(61 )92063-3

21. Harris WH, Schiller AL, Scholler JM, Freiberg RA, Scott R. Extensive localized bone resorption in the femur following total hip replacement. J Bone Joint Surg Am. 1976;58(5):612-8.

22. Maloney WJ, Jasty M, Rosenberg A, Harris WH. Bone lysis in well-fixed cemented femoral components. J Bone Joint Surg Br. 1990;72(6):966-70.

23. Jones LC, Hungerford DS. Cement disease. Clin Orthop Relat Res. 1987;(225):192206.

24. Cooper RA, McAllister CM, Borden LS, Bauer TW. Polyethylene debris-induced osteolysis and loosening in uncemented total hip arthroplasty: a cause of late failure. 
J Arthroplasty. 1992;7(3):285-90. https://d oi.org/10.1016/0883-5403(92)90050-z

25. Zhu YH, Chiu KY, Tang WM. Polyethylene wear and osteolysis in total hip arthroplasty. J Orthop Surg (Hong Kong). 2001;9(1):91-9. https://doi.org/10.1177/23 0949900100900117

26. Røkkum M, Brandt M, Bye K, Hetland KR, Waage S, Reigstad A. Polyethylene wear, osteolysis and acetabular loosening with an HA-coated hip prosthesis: a follow-up of 94 consecutive arthroplasties. J Bone Joint Surg Br. 1999;81(4):582-9. https://doi.org/ 10.1302/0301-620x.81b4.8715

27. Pontificia Universidad Javeriana. Facultad de Medicina, 75 años entregando el corazón. Bogotá: Editorial Javeriana; 2017.

28. Fundación Cosme y Damián. Banco de Huesos y Tejidos. Historia [internet]. Bogotá; s. f. Disponible en: http://www.cyd bank.org/historia.php

29. Navas J, Cadena E, González JC, Zayed G. Remplazo total de cadera híbrido. Rev Colomb Ortop Traumatol [internet]. 1992;6(2):125-37. http://www.sccot.org.co /pdf/RevistaDigital/1992/Vol6N2/125-137. $\mathrm{pdf}$

30. Pérez R, Garavito L. Guía de práctica clínica para el tratamiento de la osteoartritis de cadera. Bogotá: Hospital Universitario San Ignacio; 2013.

31. Pivec R, Johnson AJ, Mears SC, Mont MA. Hip arthroplasty. Lancet. 2012;380:1768-77. https://doi.org/10.1016/ S0140-6736(12)60607-2

32. Okafor L, Chen AF. Patient satisfaction and total hip arthroplasty: a review. Arthroplasty. 2019;1(6). https://doi.org/10. 1186/s42836-019-0007-3

33. Ng CY, Ballantyne JA, Brenkel IJ. Quality of life and functional outcome after primary total hip replacement: a fiveyear follow-up. J Bone Joint Surg Br. 2007;89(7):868-73. https://doi.org/10.1302 10301-620X.89B7.18482

34. Walker RP, Gee M, Wong F, Shah Z, George M, Bankes MJ et al. Functional outcomes of total hip arthroplasty in patients aged 30 years or less: a systematic review and metaanalysis. Hip Int. 2016;26(5):424-31. https ://doi.org/10.5301/hipint.5000376

35. Evans JT, Evans JP, Walker RW, Blom AW, Whitehouse MR, Sayers A. How long does a hip replacement last? A systematic review and meta-analysis of case series and national registry reports with more than 15 years of follow-up. Lancet. 2019;393(10172):647-54. https://doi.org/1 0.1016/S0140-6736(18)31665-9

36. Premkumar A, Sheth NP. Projected volume of primary total joint arthroplasty in the U.S., 2014 to 2030. J Bone Joint Surg Am. 2018 Sep 5;100(17):1455-60.

37. Sugano N. Computer-assisted orthopaedic surgery and robotic surgery in total hip arthroplasty. Clin Orthop Surg. 2013;5(1):1-9. https://doi.org/10.4055/cios .2013 .5 .1 .1

38. Illgen RL, Bukowski BR, Abiola R, Anderson P, Chughtai M, Khlopas A, et al. Roboticassisted total hip arthroplasty: outcomes at minimum two-year follow-up. Surg Technol Int. 2017;30:365-72.

39. Karunaratne S, Duan M, Pappas E, Fritsch B, Boyle R, Gupta S, et al. The effectiveness of robotic hip and knee arthroplasty on patient-reported outcomes: A systematic review and meta-analysis. Int Orthop. 2019 Jun;43 (6):1283-95. https://doi.org/10.1007 /s00264-018-4140-3

40. Kayani B, Konan S, Ayuob A, Ayyad S, Haddad FS. The current role of robotics in total hip arthroplasty. EFORT Open Rev. 2019;4(11):618-25. https://doi.org/10.1302 /2058-5241.4.180088 